\title{
Multiculturalidad en Contextos Educativos y de Desarrollo: Relevancia de Variables Psicosociales
}

\section{Antonio Fernández Castillo}

Departamento de Psicología Evolutiva y de la Educación

Facultad de Ciencias de la Educación, Universidad de Granada

\section{España}

afcastil@ugr.es 


\section{Resumen}

En el presente trabajo se analiza la posible relación entre diversos tópicos ampliamente estudiados por la Psicología Social y algunas dificultades inherentes a la diversidad cultural cada día más frecuente en nuestros contextos educativos. Se analiza en qué medida diversas variables de carácter psicosocial pueden explicar actitudes y comportamientos prejuiciosos, que incluso podrían llegar a explicar en algunos casos la aparición de manifestaciones racistas o xenófobas.

Partiendo de un somero análisis de las distintas concepciones y posibilidades de la interacción social multicultural, iremos revisando conceptos como estereotipo, prejuicio o discriminación, para desembocar en el análisis del prejuicio racial que podría estar detrás de la actitud xenófoba en la infancia y el deterioro en las interacciones infantiles. Igualmente será de nuestro interés el estudio del concepto de estigmatización social al que muchos niños orígenes culturales heterogéneos, están sometidos.

Tras esta delimitación conceptual, pasaremos en una segunda parte del trabajo a la revisión de diversas alternativas y planteamientos actuales en relación con la educación intercultural, desde una aproximación aplicada. Se examinan cuestiones cruciales asociadas a la diversidad educativa en ambientes multiculturales derivados de la presencia cada vez mayor de niños procedentes de heterogéneos orígenes culturales en nuestras aulas.

Palabras Clave: Diversidad educativa; interculturalidad; educación multicultural; prejuicio social; discriminación, etigmatización social. 


\section{Introducción}

En los últimos años la intensa afluencia migratoria desde múltiples orígenes geográficos a nuestro país, ha dado lugar a la presencia, cada vez mayor, de una heterogénea diversidad lingüística, religiosa, cultural e incluso étnica en nuestra sociedad. Esta diversidad no ha tardado en trasladarse a nuestros centros educativos, generando una situación que el sistema educativo no puede dejar de afrontar.

La afluencia de individuos con un origen cultural heterogéneo y desigual, no sólo implica la incorporación a nuestro conjunto social de nuevos valores, comportamientos e historias propias, sino que suponen, en el caso de las instituciones sociales, tener que dar respuestas eficientes a realidades personales nuevas y a veces discordantes.

En este panorama surgen planteamientos teóricos controvertidos alrededor de conceptos nuevos como serían el caso de la multiculturalidad o la interculturalidad. Incluso el concepto de diversidad cultural es susceptible de análisis y polémica teniendo en cuenta que el propio concepto de cultura ha sido puesto en tela de juicio. Tengamos en cuenta, como afirma Camilleri (1985), que "ningún individuo se encuentra con la cultura en sí, sino con "portadores de cultura" que jamás tienen la totalidad intacta de la cultura atribuida a su grupo, sino solamente la combinación que cada uno, para sí, ha estructurado" (p. 45).

En ese sentido la cultura podría entenderse como patrimonio de los grupos humanos que los particulariza y los diferencia entre sí (Kroeber y Kluchon, 1952), y caracterizarse siguiendo a Muñoz Sedano (1997) por ser un conjunto de producciones, eminentemente comportamentales, que singularizan a los grupos humanos y que se transmitirían siguiendo un proceso de "herencia social" entre generaciones. Sin embargo, esta permanencia no implica la inmutabilidad de la cultura, la cual se encuentra sometida a constantes cambios por el propio grupo que la sustenta en cuanto a los significados, símbolos, normas o valores que la componen y que son asumidos por el grupo en cuestión. Diversos autores señalan igualmente, la relevancia de los procesos de interacción social para el sustento cultural, no dejándose de lado el comportamiento verbal como un componente sustancial de la misma. 
Entre las consecuencias de la incorporación social de personas con un bagaje cultural diferente a nuestra realidad socio-educativa, surge el incuestionable reto de educar en esta nueva y potencialmente diversa amalgama multicultural.

Los flujos migratorios dan lugar a procesos de interacción en los que individuos con bagajes culturales particulares interactúan con otros individuos con patrimonios culturales diferentes. En cada una de estas situaciones se presentan tesituras donde uno de estos grupos es minoritario y el otro mayoritario, generándose circunstancias ampliamente estudiadas por diversas disciplinas. De Vreede (1990), entre otros, diferencia cuatro grandes posibilidades, ampliamente conocidas, derivadas de esa confluencia grupal: asimilación, segregación, deculturación e integración. El primero de estos posicionamientos implica la uniformidad en el sistema social, de forma que los individuos de la cultura minoritaria abandonen sus patrimonios idiosincrásicos y adopten los del grupo mayoritario. La segregación, por su parte, implicaría que los dos conjuntos culturales prevalecerían conjuntamente pero en ambientes separados y con una tendencia a la reducción de las interacciones mutuas. En tercer lugar, la deculturación conllevaría la situación forzada de abandono de los contenidos culturales originarios por parte del grupo minoritario, a éste término se asociarían posiciones como el rechazo, el racismo, etc. Por último nos encontraríamos con la integración, postura que apostaría por la interacción y el intercambio entre diversos grupos culturales en posiciones de igualdad. De este posicionamiento, por el cual se inclinan las políticas sociales más vanguardistas en la actualidad, se deriva un pluralismo convivencial que implicaría la interacción inter e intragrupos, la existencia de igualitarias oportunidades sociales, educativas, económicas, etc., así como la valoración social de la identidad cultural de los grupos e individuos de culturas minoritarias (Muñoz Sedano, 1997). Paralelamente, es en el seno del pluralismo donde se ubican las actuales visiones centradas en el interculturalismo.

En el caso concreto del contexto educativo parece incuestionable que la historia reciente de los planteamientos docentes, ha apostado por ofertar respuestas a las desiguales situaciones de necesidad educativa asociada a lo que se ha dado en llamar "diversidad" y que en la práctica está suponiendo con diferencia, uno de los retos más importante a los que se enfrentan en el momento actual los docentes. Dichos planteamientos actuales, han supuesto alteraciones notables en la estructura de la forma y el fondo del proceso de enseñanza-aprendizaje de cara a que todos los alumnos, sin excepciones, dispongan de los medios adecuados para la maximización de sus capacidades personales y sociales. 
Pensemos que desde la aparición del famoso trabajo de Warnock (1978) se rechaza la concepción tradicional de que los resultados inferiores en la evaluación psicométrica suponen la base de la diversidad con la que trabajará el engranaje educativo. Se abandona la idea de que la diversidad educativa esté asociada a deficiencias, o discapacidades, adoptándose la visión de la atención a la diversidad como algo potencialmente generalizable y eventualmente extensible a todo el colectivo de alumnos. La diversidad plantea en realidad dificultades de cara a un proceso grupal como es el implicado en el abordaje académico actual y son esas dificultades las que exigirán un esfuerzo a través de medidas especiales.

La inclusión en nuestras aulas de alumnos con un bagaje cultural y comportamental diferente, con un repertorio verbal desconocido o no funcional y una interpretación de la realidad alejada de nuestros planteamientos, hace de estos niños sujetos con necesidades educativas especiales. Pensemos que a los ya consuetudinarios problemas de desmotivación y actitudes negativas hacia el aprendizaje, graves en algunos escolares, se ha sumado de un tiempo a esta parte la presencia de alumnos con deficiencias comportamentales, intelectuales, sensoriales o motoras (entre otras) y más recientemente la escolarización de alumnos de culturas minoritarias dentro de una mayoritaria. La particularidad psicosocial de esta última situación será objeto de estudio en este trabajo por cuanto la inserción de un individuo con un equipaje cultural particular que calificamos externamente como de "minoritario" en el seno de un conjunto social que le es ajeno, da lugar a la consideración de una serie de elementos de discusión y procesos ampliamente estudiados por la Psicología desde hace ya algunos años y con graves repercusiones sobre la inserción y adaptación social de estos niños.

En la primera parte del presente trabajo, analizaremos de forma sucinta algunos tópicos y procesos psicológicos básicos estudiados de forma intensa por la Psicología contemporánea y que permiten entender mejor como operan los procesos de ajuste intercultural y en mayor medida cuáles son y cómo trabajan los elementos que en la práctica vulneran y distorsionan los procesos adaptativos de interacción e integración cultural. Este análisis permitirá una aproximación al panorama específico en la infancia y nos acercará a la comprensión de actitudes sociales negativas en el contexto educativo. Concretamente analizaremos el funcionamiento de los estereotipos sociales, los prejuicios, la discriminación y la estigmatización social como conceptos clave para entender los procesos aludidos. Posteriormente, en un segunda bloque, nos centraremos con mayor concreción, en algunas de las implicaciones sus- 
tanciales de cara a las interacciones educativas, prestando atención a planteamientos aplicados y consideraciones prácticas.

\section{Diversidad social y estereotipos}

En las interacciones entre grupos suelen estar presentes de forma implícita ciertos procesos de percepción social que solemos usar para simplificar nuestras interacciones y nuestros comportamientos en el ámbito social (Tajfel y Forgas, 1981). En estos cauces de asimilación perceptiva, solemos incluir procesos de categorización social que dan lugar a todo un tópico estudiado desde hace tiempo por la Psicología, como son los estereotipos. Los estereotipos están a la base de aspectos más controvertidos involucrados en las interacciones intergrupales, algunos de ellos bastante negativos, sobre los que más adelante nos centraremos.

Según diferentes autores como Bueno y Garrido (2002), los estereotipos nos permitirían entender de forma más sencilla el ambiente social y predecir acontecimientos que habrán de producirse en el mismo. De ahí que la función perceptiva sea uno de los componentes fundamentales de estos procesos. Además de ella, el proceso de formación de categorías sociales y la confluencia de información verbal sobre determinados grupos de individuos que comparten características comunes, junto a determinadas creencias que entran de una forma o de otra a formar parte de nuestra historia personal en relación con tales grupos, terminan de caracterizar los estereotipos.

Específicamente, Leyens, Yzerbyt y Schadron (1994) definen los estereotipos como conjuntos de creencias sobre las características de grupos de personas que son compartidas en un ambiente social. Tales características estarían constituidas eminentemente por rasgos de personalidad y sobre todo por comportamientos característicos de ese grupo de personas.

Según otros autores (Huici y Moya, 1995) sólo serían compartidos realmente, y de forma más intensa, los estereotipos culturales. Es frecuente que en el caso de grupos de personas pertenecientes a otras culturas "minoritarias", se les aplique un marco perceptivo particular por parte del grupo "mayoritario" o estereotipo que tiende a regir las interacciones entre ambos grupos con diversos tipos de efectos, no siempre positivos. Una de las cuestiones de gran relevancia en relación con los estereotipos radica en el grado de error o sesgo implicado en su utilización. En ese sentido, Brighman (1971) afirma rotundamente que se trata de per- 
cepciones erróneas por al menos tres motivos. En primer lugar, porque no son adquiridos por experiencia directa con las personas sobre las que actúa el estereotipo, sino que más bien son reglas verbales e información no fundamentada ni experienciada de forma directa. En segundo lugar, los estereotipos implican siempre generalizaciones que como tales tienden a la magnificación y al sesgo interpretativo. A todo lo anterior añade, en tercer lugar, el marcado hecho de que tiendan a mantenerse, a la dificultad en su alteración o en definitiva su rigidez.

Si a pesar de ser erróneos se mantienen quizá se deba a otras funciones que pueden desempeñar para los individuos. Aparte de la función de categorización de la que ya hemos aludido y que en realidad sería de cierta ayuda a la hora de ordenar y simplificar el ambiente social a través de la intensificación de las diferencias con otros grupos de personas, podemos considerar al menos otras cuatro funciones relevantes de los estereotipos. En primer lugar, podrían ser un justificante poderoso para el desempeño de acciones dirigidas contra determinados grupos sociales (Huici y Moya, 1995). En segundo lugar, y en la línea de lo anterior, podrían justificar igualmente determinados argumentos sobre circunstancias y acontecimientos sociales altamente complejos. En tercer lugar, pueden facilitar la explicación de características y comportamientos personales y, por último, serían el sustento de lo que se ha dado en llamar "identidad social" y que sería un componente esencial del autoconcepto. En ese sentido, las verbalizaciones que hacemos sobre nosotros mismos pueden estar relacionadas con la pertenencia a determinados grupos sociales en los que nos incluimos.

\section{Estereotipo, prejuicio y discriminación}

La inclusión de determinados atributos negativos en el estereotipo de las personas de culturas diferentes o grupos minoritarios, nos conduce a considerar dos posibles desenlaces de interés: el prejuicio y la discriminación hacia esas personas. Como hemos comentado, el estereotipo podría añadir a sus funciones la confirmación y justificación de una cierta hostilidad hacia los componentes del grupo social o cultural al que no se pertenece. Lo que a su vez y seguramente, afectará a determinadas actitudes, creencias, prácticas (incluso políticas e institucionales) y un amplio rango de circunstancias en relación con estas personas.

El prejuicio se sustenta en el ámbito afectivo y es el resultado directo de prestar atención a los atributos negativos incluidos en el estereotipo y la posterior evaluación igualmente negativa de personas que por sus características se incluirían en el grupo estereotipado. El 
prejuicio se manifiesta por tanto, a través de valoraciones negativas sobre determinadas personas en virtud de su inclusión o pertenencia al grupo de personas de otra cultura. Debido a que se centra con cierta exclusividad en atributos negativos o indeseables, el prejuicio siempre tiende a ser injusto y a basarse en la sobregeneralización. (Morales y Páez, 1996). La manifestación más evidente de prejuicio en relación con individuos de grupos étnicos o culturales minoritarios es el racismo, que al igual que otro tipo de prejuicios, no son sino parte de la maquinaria ideológica que se utiliza por determinados grupos sociales para justificar actitudes y en general una atención particular hacia esos grupos minoritarios.

La discriminación como un paso más en este proceso, sería el resultado que quizá se manifieste de forma más objetiva, dado que se proyecta en forma de comportamientos negativos hacia las personas que componen el grupo social minoritario debido a su mera y supuesta adecuación a los atributos incluidos en el estereotipo. Esos comportamientos discriminatorios se ponen en funcionamiento en cualquier ámbito social, incluso institucional. Un ejemplo de los contextos en que, por ejemplo, la discriminación se pone de manifiesto, es el laboral. Y no sólo porque habitualmente se les ofrezcan trabajos mal remunerados, sin protección, en condiciones deficitarias e incluso marginales, a muchos trabajadores eficientes, sino también por la reubicación en el ámbito empresarial, la consideración de su ineficacia, etc.

\begin{tabular}{|l|l|l|l|}
\hline$\underline{\text { Proceso }}$ & Estereotipo & Prejuicio & Discriminación \\
\hline $\begin{array}{l}\text { Componente ca- } \\
\text { racterístico }\end{array}$ & Componente Cognitivo & Componente Afectivo & $\begin{array}{l}\text { Componente Comporta- } \\
\text { mental }\end{array}$ \\
\hline
\end{tabular}

Aunque los tres conceptos, estereotipo, prejuicio y discriminación se hayan frecuentemente asociados, pueden darse circunstancias en que un estereotipo negativo evolucione a positivo sin que por ello se alteren los prejuicios o los procesos discriminatorios asociados al estereotipo original (Moya y Pérez, 1990) y esta circunstancia es importante cuando trabajamos con niños, pues en definitiva hace que derivemos nuestra atención hacia las manifestaciones comportamentales y verbales de este proceso. Además no son el prejuicio y la discriminación los únicos efectos del funcionamiento del estereotipo cultural, sino que hay otras consecuencias de interés para la Psicología y con connotaciones importantes de cara al desarrollo de procesos interculturales en las que nos detendremos a continuación. 
En el caso específico de la infancia, posiblemente la visión estereotipada sobre los niños pertenecientes a otras culturas, sea un estereotipo más poderoso y pernicioso que otros. No sólo por las potenciales implicaciones en el ámbito académico y de relaciones interpersonales, sino también porque la visión negativa de estos niños puede afectar a procesos autoperceptivos, conduciendo incluso hasta una autoestima reducida. El asumir el estereotipo puede justificar para el individuo las pérdidas educativas, sociales, comportamentales, cognitivas e intelectuales, etc., a que pueda estar sometido, y lo que es todavía peor, contribuir incluso a la aceleración de procesos negativos como inadaptación social, fracaso escolar, problemas de comportamiento, etc. Posiblemente la aceptación e interiorización del estereotipo pueda ser un agente limitador para la persona estereotipada, de forma que ésta podría reducir su implicación social y su actividad como consecuencia de tomar partido por los componentes atribucionales asumidos.

De hecho, determinas circunstancias (procesos negativos como inadaptación social, fracaso escolar, problemas de comportamiento, etc., ya citados) en asociación al funcionamiento del estereotipo en su dimensión interiorizada, podrían estar a la base de una reducción en las actividades y las interacciones sociales y en definitiva a una más intensa confirmación de los componentes negativos incluidos en el estereotipo.

Otro aspecto que también puede verse acuciado por el estereotipo son los procesos de atribución. En ese sentido cuando las personas de otros grupos culturales (minoritarios) cometen fallos o errores en determinadas ejecuciones, tales incorrecciones serían más atribuidas a características negativas incluidas en el estereotipo, que a factores ambientales, por ejemplo. Dando esto lugar tras el paso del tiempo, por ejemplo a reducción de interacciones y lo que en el caso de la infancia es todavía más grave, a la falta de oportunidades para el desarrollo.

\section{La estigmatización social}

Hemos visto que poseer alguna discapacidad o pertenecer a algún grupo cultural minoritario o étnico puede ser objeto de actitudes prejuiciosas. Pero el efecto puede ser más intenso o amplio si tomamos otros ejemplos como el tener demasiado peso, demasiada edad, por pertenecer a un grupo con orientación sexual minoritario, etc. Si prestamos atención al 
individuo que presenta esas características o pertenece a esos grupos, en ocasiones podemos encontrar un efecto particular que los investigadores han denominado estigma. Este término, utilizado por primera vez y hace ya bastante tiempo por Goffman (1963), hace referencia a toda característica o atributo de una persona que presentará connotaciones negativas y "amplio descrédito" por parte de los demás, ubicándose la persona estigmatizada en una posición social anormal. Más recientemente Crocker, Major y Steele (1998) advierten de la devaluación de la identidad social por parte de la persona estigmatizada además de la relatividad contextual en el funcionamiento del estigma. Algunos autores como Goffman (1963) han clasificado los estigmas en socioculturales, físicos y psicológicos, mientras que otros han centrado su atención en el análisis de sus principales dimensiones. En este caso, Jones et al. (1984) diferenciaban seis dimensiones que caracterizan los estigmas sociales. Serían las siguientes:

- Visibilidad. Haría referencia al grado en que el estigma es evidente o puede ser ocultado. (Ej. obesidad, parálisis cerebral, credo religioso, rasgos étnicos).

- Evolución o grado de estabilidad del estigma. Hay estigmas que se reducirán o desaparecerán con el tiempo. (Ej. Permanencia temporal en silla de ruedas).

- Disruptividad. Se referiría al grado de alteración en las relaciones interpersonales debida al estigma. (Ej. Esquizofrenia.)

- Características estéticas. Hay situaciones origen de estigmatización con implicaciones estéticas negativas. (Ej. Obesidad, deformidades, etc.).

- Controlabilidad. Esta dimensión hace referencia a la responsabilidad que la persona tiene en relación con la adquisición o mantenimiento de la estigmatización. (Ej. Obesidad por un problema endocrino o por una ingesta excesiva más inmovilidad).

- Peligro. Se referiría a aquellas situaciones estigmatizantes que pueden generar situaciones de riesgo o amenaza para otros. (Ej. Enfermedades infecto-contagiosas como el SIDA).

Es conveniente recalcar que el estigma supone en el individuo estigmatizado la existencia de algún tipo de información específica, atributo o "señal percibida o inferida" (Jones et al. , 1984) que lo aleja de lo normal en sentido negativo mientras que el estereotipo supone eminentemente un conjunto de creencias y procesos perceptivos (potencialmente erróneos) sobre personas por su mera pertenencia a un grupo social al cual se le adscribe un conjunto de características y atributos. Todavía hoy el término estigma conserva en cierto sentido algo de 
su utilización originaria en la antigua Grecia, donde el estigma denominaba la marca que se les hacía a los esclavos que habían intentado huir o a ciertos criminales. El estigma identificaba negativamente a ciertos individuos a los que se evitaba socialmente.

Otra cuestión interesante en relación con el funcionamiento del estigma es el propio proceso en sí de estigmatización y en este sentido podemos diferenciar entre dos puntos de vista diferentes, el de la persona que estigmatiza y el de la persona estigmatizada. En el primer caso, para que se produzca la estigmatización han de confluir varios pasos en ese proceso:

- Existencia de un poderoso estereotipo negativo en relación, en nuestro caso, con un grupo social o cultural.

Es interesante señalar que aunque estereotipos y estigmas están asociados, no son sinónimos como ya hemos visto, e incluso, al hilo de investigaciones recientes (Biernat y Dovidio, 2000), podemos encontrar procesos de estigmatización en ausencia de estereotipos. Según estos autores en el caso de la estigmatización de individuos por su pertenencia a grupos socio-culturales minoritarios, el estereotipo sí desempeñaría un papel importante sobre todo como soporte de la estigmatización. Este papel o esta asociación con el estereotipo no sería tan importante en el caso de otros procesos de estigmatización asociados sobre todo al padecimiento de enfermedades, malformaciones, minusvalías, etc.

a Ausencia de estabilidad inicial en la información valorativa hacia el estigmatizado.

Es frecuente encontrar sentimientos encontrados y ambigüedad en las valoraciones hacia personas estigmatizadas, oscilando desde sentimientos positivos a negativos. Por ejemplo la ausencia de responsabilidad sobre la aparición del estigma genera sentimientos positivos. Igualmente, cuando los individuos estigmatizados, miembros de un grupo social minoritario, se comportan positivamente tienden a ser sobrevalorados, al contrario de lo que ocurre cuando se comportan negativamente (Pérez, 1996).

口 Presencia de síndrome de activación o ansiedad en las relaciones interpersonales con personas estigmatizadas. 
Algunos ejemplos de interacciones diferentes con personas estigmatizadas incluirían mayor distancia interpersonal en las interacciones verbales, escaso contacto visual, conversación breve, interacciones pobres, tendencia a acabar pronto la conversación, evitación, etc. Posiblemente en estas reacciones, se vean implícitas potenciales contingencias negativas para la persona no estigmatizada, percepción de ridículo, rechazo, miedo, etc.

a Falta de concordancia entre las actitudes y valores y los comportamientos hacia individuos estigmatizados.

Sería posible encontrar actitudes y valores sutilmente expresadas en conducta verbal positiva referida a personas estigmatizadas y posteriormente comportamiento manifiesto más negativo con respecto a esas mismas personas estigmatizadas. Esta posibilidad ha sido asociada más intensamente con el prejuicio racial (Crosby, Bromley y Save, 1980). Actualmente encontramos en nuestro ámbito social grandes defensas de la interculturalidad y un penetrante patrimonio ideológico que defiende la igualdad, la integración, etc., que coexiste al mismo tiempo con manifestaciones comportamentales negativas y prácticas discriminatorias que vendrían a ejemplificar esta tesitura.

Junto a lo anterior, sin embargo, por parte de la persona estigmatizada existen otros procesos interesantes que se insertan en el proceso de estigmatización, en los cuales nos detendremos a continuación.

- En primer lugar, podríamos considerar la toma de conciencia sobre el funcionamiento de un estereotipo social que subsiste en el grupo al que se pertenece.

Es frecuente que las personas que pertenecen a un grupo social sepan los atributos incluidos en el estereotipo que se les aplica, aunque no compartan o no entiendan como cierta esa información (Jones et al., 1984). La persona estigmatizada podría asumir en ciertas ocasiones como cierto el contenido del estereotipo, sirviendo como herramienta de justificación y racionalización de sus fracasos o sus comportamientos en determinadas circunstancias.

- Existencia de experiencias en relación con prejuicios y discriminación relacionadas o derivadas del funcionamiento del estereotipo social y toma de conciencia de la información prejuiciosa que los demás tienen sobre el grupo al que se pertenece. 
Existe evidencia contradictoria sobre esta cuestión, pues mientras para algunos autores las personas estigmatizadas no son siempre conscientes de ser víctimas de discriminación, para otros las personas víctimas de estigma suelen ser altamente sensibles a situaciones potencialmente discriminatorias (Operario y Fiske, 2001). En general, el haber estado expuesto a situaciones negativas de interacción social y a experiencias aversivas y discriminatorias, generan consecuencias negativas en la persona estigmatizada, como por ejemplo descenso en su autoestima, visión negativa sobre las interacciones sociales, consecuencias en su adaptación e integración social, etc.

a Si existen episodios de discriminación o que se interpretan como tales, el individuo estigmatizado muestra indeterminación o confusión en al atribución de tales episodios.

La incertidumbre puede ser una circunstancia presente en la explicación de ciertos acontecimientos, los cuales no pueden ser explicados con certeza como derivados del funcionamiento del prejuicio social asociado a la estigmatización en la que el individuo se encuentra inmerso o como derivados de causas externas e independientes. Esta ambigüedad atribucional ha sido asociada con diversas variables como pérdida de bienestar, padecimiento de mayor amenaza, decremento de la autoestima, sensación de falta de control situacional, ansiedad, etc.

Los procesos de estigmatización podrían implicar una cuestionada utilidad para las personas que estigmatizan. En esa línea por ejemplo, algunos autores señalan que la estigmatización de otros podría ser una fuente de incremento de la autoestima propia (Wills, 1981), en el sentido de que quién estigmatiza se siente superior a la persona estigmatizada.

El funcionamiento de la estigmatización y los estereotipos, podría servir como componente de racionalización o justificante del sistema social y la legitimidad del orden sociocultural. Las personas de mayor estatus tenderían a estigmatizar a las de menor estatus, justificando así sus privilegios y racionalizando y justificando de esa forma su posición. El sistema que beneficia a ciertos grupos sería entendido como justo por esos grupos. Algunas ideologías se apoyan en estereotipos y procesos de estigmatización que justifican la posición social de cada conjunto de individuos (Sidanius y Prato, 1993). De esta perspectiva se derivaría el hecho de la aceptación del estigma por parte de la persona estigmatizada, la cual tendería a pensar que efectivamente el sistema social es justo pues cada uno desempeña su papel. 
Otra función potencial sería la que se relaciona con la identidad social (Tajfel y Turner, 1979), de este posicionamiento teórico se derivaría que la comparación con personas de otros grupos sociales de inferior situación, hace sentir al grupo de pertenencia como superior y en este transcurso, los procesos de estigmatización jugarían un papel importante. Por último podríamos hacer referencia a la reducción de algunas tipologías de ansiedad como consecuencia del uso del estigma y los estereotipos socio-culturales (González, Cortes y Rodríguez, 2000).

Para el caso concreto de la infancia, hay una cuestión de capital importancia. Esta se relaciona con la adquisición y mantenimiento de todos estos procesos psicosociales. En la infancia, posiblemente la articulación de los estereotipos y los procesos derivados de los mismos, así como las implicaciones asociadas con la estigmatización social, se ponen en funcionamiento a través de cualquier proceso de adquisición comportamental (Fernández Castillo, 2002) y eminentemente por la exposición a modelos y por la asimilación de información verbal relacionada con los sujetos objeto de estigmatización. Esta consideración nos conduce a pensar que los procesos educativos que se lleven a cabo en la infancia, tendrán una gran responsabilidad en la génesis de procesos que faciliten o impidan el funcionamiento de los postulados asumidos en la interculturalidad.

Para concluir este apartado, señalar que un gran conjunto de estudios ha relacionado la estigmatización con otras variables de diversa importancia psicológica como depresión, autoestima, satisfacción, rendimiento académico, etc., encontrando resultados que hacen del estigma y de las consecuencias del uso de estereotipos, una variable a tener en consideración en el estudio de todos estos tópicos de la Psicología contemporánea.

A lo largo del apartado siguiente estudiaremos algunas de las repercusiones socioeducativas que conllevan las consideraciones revisadas, de cara, no sólo a la evitación de la ocurrencia de las manifestaciones perniciosas comentadas, sino también y sobre todo, a la potenciación de situaciones de interculturalidad educativa real. Nos centraremos, por tanto, a continuación en aquellas derivaciones aplicadas que se desprenden de las aportaciones e investigaciones llevadas a cabo sobre los temas a los que hemos prestado atención. 


\section{Consideraciones psicosociales para la educación intercultural}

Los planteamientos actuales en relación con la educación intercultural asumen una serie de postulados básicos a través de los cuales se pueda promover la plena integración y el funcionamiento igualitario en las aulas. Entre los objetivos teoréticos implicados se destacan la potenciación de valores como la tolerancia, el respeto, el pluralismo, entre otros, bajo la premisa de que todos los alumnos con independencia de su origen socio-cultural, tienen derecho a participar de un sistema de enseñanza-aprendizaje que garantice el crecimiento personal en la máxima expresión posible. Podríamos entender como necesarias otras consideraciones adicionales que desde hace ya algún tiempo se vienen trabajando en alumnos con necesidades educativas especiales (Fernández Castillo, 2001), como por ejemplo y entre otras cuestiones:

- Fomentar la inclusión funcional en grupos normalizados en la medida de lo posible,

- Especial atención a las particularidades que puedan presentarse en cada caso, además o asociadas a circunstancias de necesidades educativas especiales.

- El reconocimiento y el respeto de las diferencias individuales (en este caso eminentemente socio-culturales).

- Evitar la utilización de diagnósticos o etiquetas que permitan la delimitación de una persona en función de las mismas.

․ Fomento de procesos de comunicación eficiente.

a Y por supuesto llevar a cabo las necesarias modificaciones en los objetivos educativos, la metodología puesta en funcionamiento, las actividades desarrolladas o los sistemas de evaluación a los que se recurra, entre otras consideraciones a incluir en una eventual adaptación curricular.

A todo lo anterior, y en consonancia con los puntos anteriores de este trabajo, tendríamos que añadir que todas estas consideraciones habrían de ser complementadas con una atención exhaustiva a los procesos a los cuales hemos hecho referencia. El proceso de enseñanzaaprendizaje ha de contemplar la atención al desarrollo de estereotipos, prejuicios y potenciales situaciones de discriminación que se deriven de ahí, e incluso a la aparición de situaciones de estigmatización social en el contexto educativo, sobre esta tesitura nos centraremos a continuación. 
En primer lugar, se habrán de tener en cuenta objetivos de intervención que incluyan la rotura del ciclo del prejuicio ya comentado. Está claro que en la infancia la exposición a la información prejuiciosa, contenidos verbales y exposición a modelos (parentales o de otra naturaleza) que defiendan o amparen los aspectos y atributos incluidos en prejuicios socioculturales, es la primera vía de adquisición de este tipo de esquemas cognitivos con los que ulteriormente el sujeto analiza la realidad. Dicho en otras palabras, habremos de educar a no entender de forma generalizada e igualitaria a los que son distintos a nosotros. Habitualmente el sistema social, a través fundamentalmente de los padres (la familia), los educadores formales e informales y los medios de comunicación, fomenta el funcionamiento de los estereotipos. Se ha de tener por tanto actitudes tolerantes, abiertas, de aceptación, etc., por parte de quienes interactúan formativamente con la infancia de cara a evitar la "transmisión" de visiones estereotipadas. Sólo de esta forma podremos evitar la aparición de los dos ulteriores componentes, más destructivos, asociados al estereotipo, esto es el surgimiento del componente afectivo asociado al mismo o prejuicio y lo que resulta más grave aún, la aparición de comportamientos derivados y que dan lugar a situaciones de discriminación.

En segundo lugar, una vez tenido en cuenta lo anterior y si ya se encuentra instaurado el estereotipo en el repertorio comportamental infantil, manifestándose fundamentalmente en conducta verbal, aún se podría recurrir a la potenciación del contacto intergrupal directo como medida eficaz de contrarresto de las visiones estereotipadas y las actitudes prejuiciosas. Según diversos autores como Pettigrew (1981) parece evidente que el incremento de las relaciones interpersonales entre grupos diferenciados de individuos facilitaría el reconocimiento de similitudes entre ellos. Pensemos que uno de los efectos del estereotipo es la visión de los individuos incluidos en el otro grupo (cultural en nuestro caso) como atribuidos por las mismas características, este sesgo de generalización se debilitaría cuando se entra en contacto personalizado con los miembros de ese grupo. Adicionalmente al efecto de erosión del estereotipo y el prejuicio comentado, también es sostenible que una de las formas más potentes de modificación de estereotipos (los cuales como ya vimos son bastante resistentes al cambio) radica en el hecho de confrontarlos con información no concordante con sus contenidos. Las excepciones y las informaciones opuestas a las contenidas en el estereotipo, son las variables que más hacen despreciar al estereotipo como marco de visión de un grupo social (Kunda y Oleson, 1995). Algunas variables estudiadas que remarcan la efectividad del contacto intergrupal di- 
recto incluirían la semejanza en el estatus social de los miembros de diferentes grupos que interactúan, lo que en el caso del contexto educativo formal crea una situación idónea.

En tercer lugar, se postula que las interacciones informales son más efectivas que las formales. Lo que nos conduciría al interés en la potenciación de actividades de esa índole en los contextos educativos. También se destacan como más efectivas de cara a la reducción del estereotipo y sus derivados, las actividades donde se involucran procesos de cooperación e interdependencia, es decir la colaboración para el alcance de metas derivadas exclusivamente del trabajo de grupos multiculturales en nuestro caso.

En cuarto lugar, habría que destacar la necesaria existencia en el seno de los grupos multiculturales de unas normas de funcionamiento que permitan la igualdad para todos los involucrados en la actividad grupal. Por supuesto y de forma obvia, los participantes no habrían de dar crédito a los contenidos incluidos en el estereotipo o dicho de otra forma, tendrían que partir de una posición de rechazo de dichos contenidos. Estas condiciones asociadas a la interacción entre personas pertenecientes a diferentes grupos socio-culturales, garantizarían la disminución prejuiciosa y debilitarían el funcionamiento del estigma a través de la aparición de una identidad común endogrupal (Cook, 1985; Gaertner et al.,1993; Riordan, 1978).

Es importante fomentar en los niños el hecho de que presten atención cuidadosamente a los demás, de forma que se incremente la percepción de la individualidad de los miembros pertenecientes a un grupo cultural diferente y no se caiga en la homogeneización de todos los miembros. En ese sentido, serían más importantes esas características y atributos personales que el hecho de su mera adscripción a un grupo social diferente al nuestro. Cuando forzamos o motivamos la exactitud en la descripción de los individuos, se reduce la credibilidad del estereotipo. (Neuberg, 1989).

Además no hemos de olvidar prestar atención a los factores que afectan a la consecución de determinados resultados que obtienen individuos pertenecientes a grupos "minoritarios". A menudo sólo se percibe el resultado y no a los procesos que lo han afectado (Allison, Worth y King, 1990). Por ejemplo, un alumno de una cultura diferente con dificultades para 
entender el castellano, puede presentar problemas en la realización de determinadas actividades escolares y procesos evaluativos y derivar hacia un fracaso académico, contribuyendo este último hecho a la potenciación de una posible situación personal de estigmatización.

Pensemos que la defensa tanto de situaciones de educación multi como intercultural implica la aceptación de un pluralismo cultural en el seno del cual las diferencias culturales no solo se aceptan y se respetan, sino que además se valoran positivamente y se integran en todos los procesos de interacción grupal e individual.

Además de las consideraciones precedentes asociadas a los estereotipos y otros conceptos relacionados, si de verdad queremos potenciar la interculturalidad en el seno de los procesos psicoeducativos, habremos de tener en cuenta entre otras las consideraciones siguientes (Banks, 1994).

- Habrán de promoverse metodologías, actividades y, en general, actitudes y posicionamientos que reduzcan situaciones de visión estereotipada y discriminación de alumnos susceptibles de ser adscritos a grupos culturales minoritarios. En ese sentido, resulta imprescindible, como ya hemos comentado, el favorecer estrategias que reduzcan la aparición y el funcionamiento pernicioso de los prejuicios hacia alumnos "diferentes".

- Todos los alumnos habrían de recibir formación en actitudes, conocimientos y habilidades que les permitiesen desarrollar hábitos comportamentales funcionales dentro de su cultura de referencia. Con este tipo de iniciativas, el alumno desarrollaría estrategias que le permitirían funcionar exitosamente en su cultura de origen.

- Con independencia del punto anterior, y de forma paralela, se entendería como fundamental la capacitación y la adquisición de habilidades involucradas en los propios procesos de enseñanza-aprendizaje donde el alumno se ve inmerso. Obviamente los objetivos curriculares que se persigan se verán dificultados si el alumno carece de determinadas habilidades que se entienden como prerrequisitas.

- Los centros educativos habrían de ofrecer alternativas culturales involucradas en sus planteamientos. Quizá esta sea una de las cuestiones más dificultosas en la medida que chocará con la tendencia etnocéntrica de los propios docentes, aparte de la falta de formación y otros 
problemas. Vencer el "hispanocentrismo" implicaría ofertar contenidos pertenecientes a otras culturas (estilos de vida, artes, valores, etc.) entre los objetivos curriculares normalizados.

- Por último, en este conjunto general de consideraciones, todo lo anterior nos conduciría a la promoción genérica de la comprensión de otras culturas, pues es a través de esa comprensión donde los alumnos podrán asimilar los valores de su propia cultura y la idiosincrasia de otras alternativas culturales. A partir también de este conocimiento podrán trabajarse los valores comentados con anterioridad entre los que se incluirían el respeto y la valoración mutua.

En relación con los déficits comunicativos y de lenguaje presentes de manera habitual en alumnos de grupos socio-culturales, y entendiendo que el comportamiento verbal puede interpretarse no sólo como el principal escollo para las relaciones interpersonales, sino también para el desarrollo de objetivos educativos Briggs, (1991, pp. 11-14) propone, entre otras, las consideraciones que se agrupan en el cuadro siguiente.

- Usar gestos

- Usar la interacción visual

- Escribir la información relevante

- Comprobar con frecuencia la comprensión

- Proponer múltiples actividades prácticas

- Utilizar el aprendizaje cooperativo y otros tipos de aprendizaje en grupo.

- Centrarse en la comunicación

- Discutir las estrategias de aprendizaje

- Utilizar la experiencia de los alumnos

- Disponer de varias herramientas de evaluación

- Medir el tiempo de reacción

- Utilización de la lengua materna de los alumnos

- Proporcionar ayuda a los alumnos en el aula

Recordemos igualmente que el lenguaje adquiere una de sus principales funciones en el ámbito de la comunicación y que ambas variables (lenguaje y comunicación) se relacionan de una forma crucial con el desarrollo de interacciones sociales eficientes. 
En esa línea, entre las estrategias que potencian el funcionamiento multilingüe en el aula, podrían recoge las siguientes (Adaptado de Cummins, 1989, pp. 113-114).

- Animar a los alumnos a que utilicen su lengua materna en clase.

- Creación de varios grupos culturales dentro de la escuela y dar la bienvenida a los alumnos que hablan distintas lenguas.

- Contar con libros y materiales escritos en distintas lenguas para trabajar en clase.

- Generar oportunidades a los alumnos para que hablen de las costumbres y los valores de su cultura de referencia.

- Contar con personas que puedan servir como tutores de estos alumnos y que hablen su misma lengua.

- Incorporar información en distintas lenguas en diversas actividades en la escuela.

- Adornar el centro con objetos, dibujos y fotografías de diferentes culturas.

- Utilizar signos bilingües y multilingües.

- Crear unidades didácticas en las que se incorpore la lengua materna de los distintos alumnos.

- Pedir a los alumnos que contribuyan a diversas actividades de la escuela con contribuciones originarias de su cultura de pertenencia.

- Dar oportunidades a los alumnos para que formen asociaciones y clubes en los que se hable su lengua materna y se realicen diversas actividades.

- Implicar a los padres en las actividades de la clase y el centro.

- Invitar a personas que utilizan esa lengua como segundo lenguaje a que la usen como lengua primaria en determinados contextos, como por ejemplo asambleas, reuniones, etc.

- Invitar a personas de esa comunidad para que interactúen con los alumnos tanto en un contexto formal como informal.

La potenciación de la comunicación en el aula ha de ser un instrumento que colabore en la creación de un clima positivo en clase, lo que junto a la utilización de recursos y estrategias educativas adecuadas, podrá permitir a los docentes estructurar y diseñar respuestas particulares a situaciones individuales de necesidades educativas específicas y únicas. 


\section{Discusión}

Cualquiera de las previsiones que podamos consultar sobre la cuestión señalan que es de esperar a corto y medio plazo, si las pautas y los flujos migratorios actuales se mantienen, la paulatina presencia de niños pertenecientes a diferentes grupos culturales en nuestras aulas. Independientemente de la concienciación del sistema educativo sobre esta situación, habrán de tomarse las medidas pertinentes que garanticen una respuesta educativa adecuada y eficiente a esta tesitura.

Cualquier cambio que observemos en el amplio espectro que se extiende desde las interacciones sociales en la infancia hasta las políticas y actitudes sociales con respecto al sistema educativo, dirigido hacia el desarrollo de sistemas virtualmente interculturales, no será efectivo ni incluso real, si no se origina paralelamente un acercamiento social en el que no se deje de lado la aceptación y un sincero deseo de igualdad entre los grupos humanos integrados por individuos con diferentes bagajes culturales.

Con independencia de las políticas sociales y educativas de turno, los educadores habrán de llevar a cabo un importante esfuerzo en el cual se incluya el trabajo de la comprensión por parte de sus alumnos de la situación social actual, de las causas de la diversidad cultural y de sus múltiples implicaciones. En ese sentido, emergen como puntos cardinales tres cuestiones primordiales a las cuales nos remiten de forma esquemática los aspectos revisados en este trabajo. De una parte, la necesaria potenciación de actitudes favorables en todos los protagonistas que guardan relación o se ven involucrados en cualquier proceso de enseñanzaaprendizaje tanto en contextos educativos formales como informales. De la otra, el fortalecimiento de la comunicación en el seno de los grupos multiculturales, como paso previo a una tercera cuestión capital, el incremento de las relaciones interpersonales e intergrupales en condiciones de aceptación e igualdad, donde estén presentes individuos con bagajes sociales, étnicos o culturales diferentes. Estas cuestiones ampliamente justificadas por la literatura psico-socio-educativa, se constituirán en garante de un proceso difícil que seguiremos afrontando en un futuro próximo, pero que sin lugar a dudas estamos abordando ya. 


\section{Referencias}

Allison, S.T., Worth, L.T., y King, M.C. (1990). Group decisions as social inference heuristics. Journal of Personality and Social Psychology, 58, 801-811.

Banks, J.A. (1994). An introduction to multicultural education. Boston: Allyn and Bacon.

Biernat, M., y Dovidio, J.F. (2000) Stigma and stereotypes. En T.F. Heatherton, R.E. Kleck, M.R. Hebl, y J.G. Hull (Eds.), The social psychology of stigma (pp. 88-125). Nueva York: Guilford.

Briggs, S.J. (1991). The multilingual/multicultural classroom. Kappa Delta Pi Record, 28, 1114.

Brighman, J.C. (1971). Ethnic stereotypes. Psychological Bulletin, 76, 15-38.

Bueno, M.R. y Garrido, M.A. (2002). Estereotipos, prejuicios y discriminación. En M. Marín, R. Grau y S. Yubero (Eds.), Procesos psicosociales en los contextos educativos. Madrid: Pirámide.

Camilleri, C. (1985). Antropología cultural y educación. París. UNESCO.

Cook, S.W. (1985). Experimenting on social issues: The case of school desegregation. American Psychologist, 40, 452-460.

Crocker, J., Major, B. y Steele, C. (1998). Social stigma. En D. Gilbert, S.T. Fiske y G. Lindzey (Eds.) Handbook of social psychology. Boston: McGraw Hill. (pp. 504-553).

Crosby, F., Bromley, S. y Save, L. (1980). Recent unobstrusive studies of black and white discrimination and prejudice: A literature review. Psychological Bulletin, 87, 546-563.

Cummins, J. (1989). A theoretical framework for bilingual special education. Exceptional Children, 56, 113-124.

De Vreede, E. (1990) What are we talking about? Plural education and Teacher education. European Journal of Teacher Education, 13 (3), 129-140.

Fernández Castillo, A. (2002). Rabietas. Cómo analizar y abordar problemillas de comportamiento en la infancia temprana. Valencia: Promolibro.

Fernández Castillo, A. (2001). Fundamentos psicopedagógicos de educación especial. Granada: Grupo Editorial Universitario.

Gaertner, S.L.,Dovidio, J.F., Anastasio, P.A., Bachman, B.A., y Rust, M.C. (1993). The common ingroup identity model: Recategorization and the reduction of intergroup bias. En W. Stroebe y H. Hewstone (Eds.) European Review of Social Psycholgoy, 4, $1-26$. 
Goffman, E. (1963). Stigma: Notes on the management of spoiled identity. Englewood Cliffs, NJ: Prentice Hall.

González de Chavez de Armas, L., Cortes Pozo, B. y Rodríguez Torres, R. (2000). El terror a la muerte y la percepción de los miembros del exogrupo. En D. Caballero et al. (Eds.), La mirada psicosociológica: grupos, procesos, lenguajes y cultura (pp. 280-285). Madrid: Biblioteca Nueva.

Huici, C. y Moya, M.C. (1995). Procesos de inferencias y estereotipos. En F. Morales (Coord.), Psicología Social. Madrid: UNED.

Jones, E.E., Farina, A., Hastorf, A.H., Markus, H., Miller, D.T. y Scott, R.A. (1984). Social stigma: the psychology of marked relationships. Nueva York: Freeman.

Kroeber, A.L. y Kluckhohn, C. (1952). Culture: a critical review of concepts and definitions. Cambridge. Peabody Museum of Archeology and Ethnology.

Kunda, Z., y Oleson, K.C. (1995). Maintaining stereotypes in the face of disconfirmation: Constructing grounds for subtyping deviants. Journal of Personality and Social Psychology, 68, 565-579.

Leyens, J. Yzerbyt, V.Y. y Schadron, G. (1994). Stereotypes and social cognition. Londres: Sage.

Morales, J.F., y Páez, D. (1996). Estereotipos, discriminación y relaciones intergrupos en España y en Lationoamérica. En R.Y. Bourhis y J.P. Leyens (Eds.), Estereotipos: discriminación y relaciones entre grupos. Madrid: McGraw-Hill.

Moya, M.C. y Pérez, C. (1990) Nuevas perspectivas en el estudio de los estereotipos de género. En P. Valcarcel y J.L. Meliá (Eds.). Métodos y técnicas de Intervención. Barcelona: PPV. (pp. 49-60).

Muñoz Sedano, A. (1997). Educación Intercultural. Teoría y práctica. Madrid. Escuela Española.

Neuberg, S.L. (1989). The goal of forming accurate impressions during social interaction: attenuating the impact of negative expectancies. Journal of Personality and Social Psychology, 56, 374-386.

Operario, D. y Fiske, S.T. (2001). Ethnic identity moderates perceptions of prejudice: Judgments personal versus group discrimination and subtle versus blatant bias. Personality and Social Psychology Bulletin, 27, 550-561.

Pérez, J.A. (1996). Nuevas formas de racismo. En J.F. Morales y S. Yubero (Eds.) Del prejuicio al racismo: perspectivas psicosociales. Cuenca: Universidad de Castilla-La Mancha. 
Pettigrew, T.F. (1981). Extending the stereotype concept. En D.L. Hamilton (Ed.), Cognitive processes in stereotyping and intergroup behavior (pp. 303-331). Hillsdale, NJ: Erlbaum.

Riordan, C.A. (1978). Equal-status interracial contact: A review and revision of a concept. International Journal of Intercultural Relations, 2, 161-185.

Sidanius, J. y Prato, F. (1993). The dynamics of dominance and the inevitability of oppression. En P. Sniderman y P.E. Tetlock (Eds.), Prejudice, politics and race in America today. Standford, CA: Standford University Press.

Tajfel, H. y Forgas, J.P. (1981). Social categorization: cognitions, values and groups. En J.P. Forgas (Ed.), Social Cognition. Perspectives in every life. Nueva York: Academic Press.

Tajfel, H. y Turner, J. (1979). An integrative theory of intergroupo conflict. En W.G. Austin y S. Worchel (Eds.),The social psychology of intergroup relations (pp. 33-47). Monterey, CA: Brooks/Cole.

Warnock.M (1978). Special Education Needs. Report of the Committee of Enquiry into Education of Handicapped Children and Young People. London: H.M.S.O.

Wills, T.A. (1981). Donward comparison principles in social psychology. Psychological Bulletin, 90, 245-271. 